\title{
KALA-AZAR MIMICKING CHRONIC LIVER DISEASE- A RARE PRESENTATION. A CASE FROM SIKKIM (NORTH-EASTERN STATE)
}

Mona Dhakal ${ }^{1}$, O. P. Dhakal ${ }^{2}$.

1. Associate Professor, Department of Medicine. Sikkim Manipal Institute of Medical Sciences and Central Referral Hospital (CRH).

2. Associate Professor, Department of Medicine. Sikkim Manipal Institute of Medical Sciences and Central Referral Hospital (CRH).

\section{CORRESPONDING AUTHOR:}

Mona Dhakal.

Associate Professor, Department of Medicine,

Sikkim Manipal Institute of Medical Sciences and,

Central Referral Hospital. (CRH).

$5^{\text {th }}$ mile Tadong, Gangtok Sikkim. 737102.

E-mail: mona3dhakal@gmail.com

INTRODUCTION: Visceral leishmaniasis, also known as kala-azar, is a vector borne disease caused by the protozoan parasite, L. donovani. [1] Visceral leishmaniasis is prevalent worldwide. [2] India is one of the countries that account for an estimated 300,000 of 500,000 cases of visceral leishmaniasis (VL) occurring annually. State of Bihar is the most affected area in India, with more than $90 \%$ of the cases, [3] and to some extent in its bordering states like West Bengal and Uttar Pradesh. [4] Functional derangement of liver in visceral leishmaniasis is reported infrequently in the literature, due to this, many cases are wrongly diagnosed and treated as hepatitis. [5] Common clinical manifestations are fever (99\%), splenomegaly (99\%), anaemia (96\%), hepatomegaly (86\%), distension of abdomen (47\%), bleeding diathesis (14\%) and pancytopenia. [6] There are case reports of visceral leishmaniasis mimicking portal hypertension, and it has been suggested that screening of the disease in children presenting with chronic liver disease is important in endemic areas. [7] Certain unusual clinical and laboratory features were seen in some of the reported cases, like lymphadenopathy, nasopharyngeal growth, acute and chronic hepatic involvement and portal hypertension. [8] One case reported from India has shown visceral leishmaniasis masquerading as chronic liver disease with abdominal doppler study revealing portal hypertension and endoscopy showing grade II oesophageal varices. [9] Sikkim is not an endemic area for kala-azar and only few sporadic cases are been reported from the state.

CASE REPORT: A 48 year old male, married, farmer by occupation, permanent resident of south Sikkim, non-alcoholic, non smoker presented in medical OPD with evening rise of temperature and nights sweats for last 6 months, gradual distension of abdomen and swelling of feet, yellowish discoloration of urine, extreme lethargy for the last 3 months. There was no past history of jaundice, tuberculosis, altered sensorium, joint swelling, hematemesis, malena and blood transfusion nor any history of prior hospital admission. There is no history of travel outside Sikkim.

On examination, patient had mild temperature (99.1 ${ }^{\circ} \mathrm{F}$ ), BP 126/76 mm Hg, pulse 98/min regular, looked very pale, had mild icterus, pedal oedema of pitting nature and distended abdomen with no prominent veins. Per abdomen examination revealed enlarged liver about $7 \mathrm{~cm}$ below costal 
margin, mildly tender, soft in consistency, enlarged spleen of about $5 \mathrm{~cm}$ below costal margin and presence of ascites. Examination of cardiovascular, respiratory, genito-urinary and central nervous systems examination did not detect any abnormality.

He was admitted in general ward with provisional diagnosis of chronic liver disease with decompensation and was investigated further.

Blood reports revealed haemoglobin of $7.8 \mathrm{~g} / \mathrm{dl}$, total leucocyte count of 2,600/cumm, P54, L30, E15 and M01, platelet count of 1lakh and ESR of $68 \mathrm{~mm} / 1^{\text {st }} \mathrm{hr}$. Peripheral blood smear showed microcytic hypochromic anemia. Serum bilirubin was $3.2 \mathrm{mg} / \mathrm{dl}$ with direct bilirubin $2.0 \mathrm{mg} / \mathrm{dl}$, SGOT 96 U/L, SGPT 104 U/L, ALP 302 U/L. Total proteins 6.4.g/dl, albumin 2.2 g/dl, globulins 4.2 $\mathrm{g} / \mathrm{dl}$. PT difference was $16 \mathrm{sec}$. INR was 2.78 (pt PT 26 sec. control $10 \mathrm{sec}$.) Kidney function test with electrolyte, fasting and PP sugar were normal. Ascitic fluid analysis was transudative in nature. Aldehyde test was positive. Blood and urine culture was sterile. Routine urine and stool examination were normal. He tested negative for malaria, typhoid, hepatitis B and C and HIV. IgM HAV and IgM HEV were negative. Chest X-ray and ECG were normal.

Ultrasound of the abdomen showed enlarged liver with normal echotexture with normal gall bladder and bile ducts. Moderate enlargement of spleen was present with few collaterals at the splenic hilum with color doppler. Free fluid in abdominal cavity was also present. On upper GI endoscopy there were 2 columns of grade II esophageal varices.

In view of pancytopenia and positive aldehyde test, bone marrow aspiration was done which showed presence of extracellular LD bodies (fig 1).

Liver biopsy could not be done due to prolonged prothrombin time and unwillingness of the patient as he had painful experience during bone marrow examination.

A diagnosis of "Kala-azar with hepatitis with portal hypertension" was made. The patient was started on sodium antimony stibogluconate $20 \mathrm{mg} / \mathrm{kg} /$ day by intravenous route and later changed to deep intramuscular route as PT was deranged initially, along with hematinics and antipyretic drugs. Patient responded very well and by third week, much regression in liver and spleen size was noted clinically. His abdominal distension decreased, became afebrile, and overall improvement was seen in his general condition. His repeat investigation showed haemoglobin of $10.2 \mathrm{~g} / \mathrm{dl}$, total leucocyte count of 4600/cumm, N64, L32, E03 and M01, platelet count of 1.3 lakhs and ESR of 24 mm/1st hr. Serum Bilirubin and liver enzymes level significantly reduced and there was increase in serum proteins (Serum bilirubin $1.4 \mathrm{mg} / \mathrm{dl}$ with direct bilirubin of $1.0 \mathrm{mg} / \mathrm{dl}$, SGOT $42 \mathrm{U} / \mathrm{L}$, SGPT 54 U/L, ALP 224 U/L. Total proteins was $7.4 \mathrm{mg} / \mathrm{dl}$, albumin $3.2 \mathrm{mg} / \mathrm{dl}$ and globulins $4.2 \mathrm{mg} / \mathrm{dl}$ ). PT difference reduced to 6 sec. USG abdomen showed only mild hepato-splenomegaly. He was discharged on request at third week and was advised to continue injection for total of 28 days at nearby PHC and to review after 15 days but he reported in medical OPD after 3 months. His follow up investigations showed normal hematological profile, normal liver function test, normal prothrombin time and disappearance of grade II esophageal varices and normal USG abdomen. As all his clinical features and laboratory parameters reverted back to normal with treatment of kala-azar, preexisting chronic liver disease seemed unlikely. He was advised to review immediately if similar symptoms appeared later in view of kala-azar relapse.

DISSCUSSION: Common clinical manifestations of visceral leishmaniasis are fever (99\%), splenomegaly (99\%), anaemia (96\%), hepatomegaly (86\%), distension of abdomen (47\%), bleeding 
diathesis (14\%) and pancytopenia. [6] There are case reports of visceral leishmaniasis mimicking portal hypertension, and it has been suggested that screening of the kala-azar in children presenting with chronic liver disease is important in endemic areas. [7] Certain unusual clinical and laboratory features were seen in some of the reported cases, like lymphadenopathy, nasopharyngeal growth, acute and chronic hepatic involvement and portal hypertension. [8] One case reported from India has shown visceral leishmaniasis masquerading as chronic liver disease with abdominal doppler study revealing portal hypertension and endoscopy showing grade II oesophageal varices similar to this report. [9] Portal hypertension and cirrhosis of liver probably do not occur as a consequence of kala-azar, [10]

Portal hypertension may be an independent manifestation of visceral leishmaniasis and remain undiagnosed unless a physician maintains a high index of suspicion. [11] Liver involvement is not unusual in kala-azar, but presentation as chronic liver disease, cirrhosis or portal hypertension is rare.[12]

As patient's clinical features and laboratory parameters reverted back to normal with treatment of kala-azar, preexisting chronic liver disease seemed unlikely.

Sikkim, being an non-endemic area for kala-azar and only sporadic cases are being reported, so it was worth reporting this unusual presentation.

\section{REFERENCES:}

1. Bhunia GS, Kesari S, Chatterjee N, Kumar V, Das P. Telehealth. A perspective approach for visceral leishmaniasis (kala-azar) control in India. Pathog Glob Health. 2012 Jul; 106(3):150-8.

2. Mishra S, Shukla A, Tripathi AK, Kumar A. Visceral leishmaniasis with HIV co-infection and cervical lymphadenopathy. BMJ Case Rep. 2013 Apr; 9.

3. Perry D, Dixon K, Garlapati R, Gendernalik A, Poche D, Poche R. Visceral leishmaniasis prevalence and associated risk factors in the saran district of Bihar, India, from 2009 to July of 2011. Am J Trop Med Hyg. 2013 Apr; 88(4):778-84.

4. Sudhakar S, Srinivas T, Palit A, Kar SK, Battacharya SK. Mapping of risk prone areas of kalaazar (Visceral leishmaniasis) in parts of Bihar State, India. An RS and GIS approach. J Vector Borne Dis. 2006 Sep; 43(3):115-22.

5. Mathur P, Samantaray JC, Samanta P. High prevalence of functional liver derangement in visceral leishmaniasis at an Indian tertiary care center. Clin Gastroenterol Hepatol. 2008 Oct; 6(10): 1170-2.

6. Tanoli ZM, Rai ME, Gandapur AS. Clinical presentation and management of visceral leishmaniasis. J Ayub Med Coll Abbottabad. 2005 Oct-Dec; 17(4):51-3.

7. Albertini M, Leger I, Marty P, Gari Toussaint M, Mary C, Saint Paul MC, Mariani R. Severe visceral leishmaniasis with hepatic involvement. Diagnostic value of the immunoblotting serologic technic. Pediatrie. 1993; 48(5):377-80.

8. Naik SR, Rao PN, Datta DV, Mehta SK, Mahajan RC, Mehta S, Chhuttani PN. Kala-azar in north-western India. A study of 24 patients. Trans R Soc Trop Med Hyg. 1979; 73(1): 61-5.

9. A Prakash, NP Singh, G Sridhara, V Malhotra, A Makhija,D Garg, Anjali Pathania, SK Agarwal. Visceral Leishmaniasis Masquerading as Chronic Liver Disease. JAPI. Nov 2006; 54: 893894. 


\section{CASE REPORT}

10. Aggarwal P, Wali JP, Chopra P. Liver in kala-azar. Indian J Gastroenterol. 1990 Apr; 9(2): 135-6.

11. Rajniti Prasad, Utpal Kant Singh, O.P. Mishra, B.P. Jaiswal, Sunil Muthusami Portal hypertension with visceral leishmaniasis. Indian Pediatrics .November 2010; 47(11): 965967.

12. D.V. Datta, S. Saha, S.L. Grover, Samant A. Singh, R.N. Chakravarti, P.N. Chhuttani. Portal hypertension in kala-azar. Gut 1972; 13:147-152.

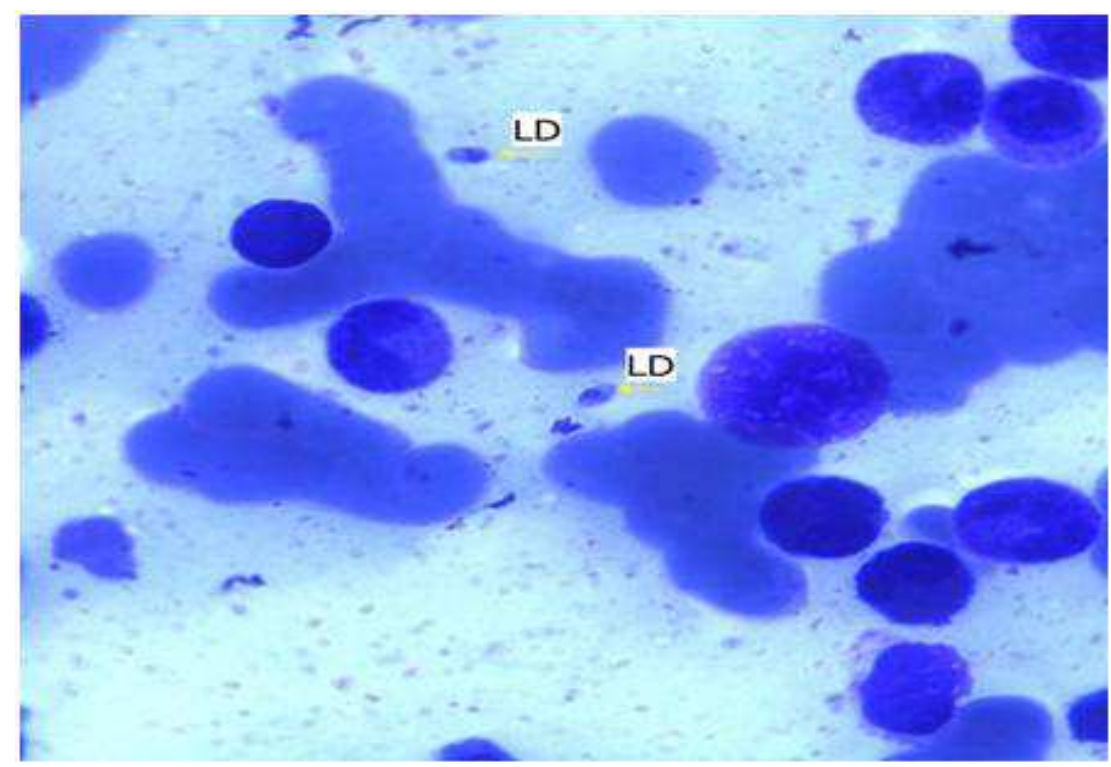

FIG 1: Showing LD bodies in bone marrow aspiration in haematoxylin and eosin stain. 